\title{
DC Pre-excitation Application in Three-phase Induction Motor Drive System
}

\author{
Sideng Hu, Student Member, IEEE Zhengming Zhao, Senior Member, IEEE, Ting Lu, Liqiang Yuan \\ Department of Electrical Engineering, Tsinghua University \\ State Key Laboratory of Control and Simulation of Power System and Generation Equipment, Beijing, China
}

\begin{abstract}
Due to the current restriction of semiconductor devices, the start-up is an extremely important process in the ASD(adjustable speed drive)system. DC pre-excitation is one of the effective solutions for increasing the starting torque and depressing the starting inrush current. However the effect of DC pre-excitation would be discounted if it could not be compatible with the induction motor drive control system. Also the stability of the DC pre-excitation application is related to its operational mode. This paper addresses the principle of DC pre-excitation and excavates its relationship with current control. The method to improve the stability of DC pre-excitation effectively shielding EMI is detailed. The relevant study has been implemented in a control system for a $55 \mathrm{~kW}$ and $160 \mathrm{~kW}$ induction motor and the simulation and experimental results validate the effectiveness of the proposed design method.
\end{abstract}

\section{Index Terms-De pre-excitation, Start-up, Anti-EMI}

\section{INTRODUCTION}

In recent years, general-purpose inverters are widely used in industry applications, such as fans, pumps, blowers, crane and hoist. PWM control and constant V/F ratio algorithms are often used in these inverters. The performance of motor star-up in the V/F controlled induction motor drive system is always receiving wide attention in previous literatures[1,2,4].The control objective in start-up is to achieve the maximum torque to amp ratio. In spite of extensive effort, the simple and effective method for the synthesis control of torque and current in $\mathrm{V} / \mathrm{F}$ controlled high power motor drivers remains a challenge nowadays. One of the existed solutions is to increase the devices allowance, but this would result in high cost especially in high power applications. There are also optimal methods to achieve better start-up performance in other ways[3,6], however they are not suitable for the induction motor drive system thus the effect is discounted in real cases.

DC pre-excitation is an effect method for the ASD system to increase the starting torque and depress the initial current in start-up process. The main characteristic in DC pre-excitation process is that the electromagnetic fields is established at set value by a closed-loop current controller before the motor starts up[5].The previous literatures on DC pre-excitation are focused on the preexcitation process. No transient process between start and acceleration process is considered and the design of closed-loop control system for excitation current in high power driver applications is neglected. This paper presents the analysis for the transient process between

Project Supported by National Natural Science Foundation of China (50737002, 50707015). start and acceleration process and the approaches to enhance the effect of closed-loop control for excitation current in high power driver applications. The relevant study has been incorporated in adjusting speed drive systems for a $55 \mathrm{~kW}$ and $160 \mathrm{~kW}$ induction motors. The simulation and experimental results validate the effectiveness of the proposed design method.

This paper is conducted to find potential factors in DC pre-excitation. The guideline of this paper is the exploration of the transient process of DC pre-excitation. In section 2, the principle of DC pre-excitation is introduced and the transient process between start and acceleration process is analyzed. In section 3 and 4, new aspects of DC pre-excitation and analysis are discussed, including the solution for improving current control in DC pre-excitation which has not been discussed in previous literatures. The simulation and experimental results validate the effectiveness of the proposed design method.

\section{PRINCIPLE OF THE DC PRE-EXCITATION}

DC pre-excitation technology establishes the electromagnetic fields in an induction motor before the motor starts up. According to the theory of vector control, we get:

$$
\begin{gathered}
\psi_{r d}=\frac{L_{m}}{1+\tau_{t} p} i_{s d} \\
T_{e m}=p_{n} \frac{L_{m}}{L_{r}} i_{s q} \psi_{r d}
\end{gathered}
$$

Neglecting the influence of the dynamic process, we can get the electromagnetic torque, that is:

$$
\begin{aligned}
& i_{s}=\sqrt{i_{s d}^{2}+i_{s q}^{2}}=\sqrt{\left(\psi_{r d} \frac{1+\tau_{r} p}{L_{m}}\right)^{2}+\left(T_{e m} \frac{L_{r}}{p_{n} L_{m} \psi_{r d}}\right)^{2}} \geq \sqrt{2 \frac{L_{r}}{p_{n} L_{m}^{2}} T^{*}{ }_{e m}} \\
& \text { If and only if } \psi_{r d}=\sqrt{\frac{L_{r} T_{e m}}{p_{n}}} \text {, the current can reach }
\end{aligned}
$$

minimum value to attain the maximum ratio of torque to amp ratio. In DC pre-excitation the excitation current is controlled by PI-type current regulator controller to establish the expected flux flied.
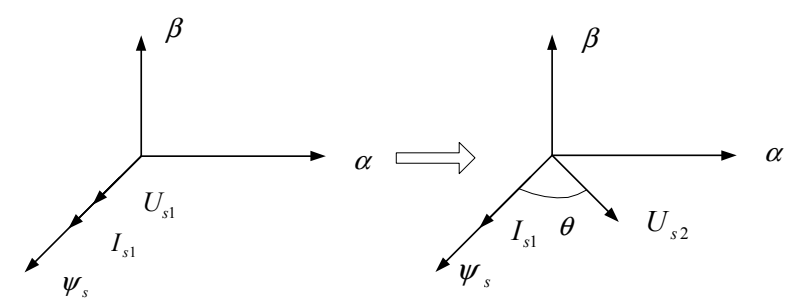
Fig.1 The vector scheme in excitation process and at the motor start moment

In the flux flied establishing process, the current curve can be described as:

$$
i_{s}(t)=\frac{U_{s}}{R_{s}}\left(1-e^{-\left(t-t_{0}\right) / \tau}\right)
$$

where $\tau$ is the time constant between stator and rotor loop when the motor is at a standstill .If $\Delta t$ is sufficiently small, the assumption of $I_{s 1}=I_{s 2}$ ( $I_{s 1}$ is the stable current in excitation process and $I_{s 2}$ is the current at the starting moment) is valid, we get:

$$
p \psi_{s}=U_{s 2}-I_{s 1} R_{s}
$$

where $U_{s 2}$ is the voltage vector at the starting moment. By cross multiplying $I_{s 2}$ at both sides, we get

$$
\begin{array}{ll} 
& p \psi_{s}=U_{s 2} \otimes I_{s 2} \\
\text { From } & T_{e}=p_{n}\left(\psi_{s} \otimes i_{s}\right)
\end{array}
$$

we get

$$
\left|\frac{d T_{e}}{d t}\right|=p_{n}\left|\left(U_{s 2} \otimes I_{s 2}\right)\right|
$$

which means at the start moment

$$
\Delta T e \approx p_{n} U_{s 2} \otimes \frac{\left(1-e^{-\left(t-t_{0}\right) / \tau}\right)}{R_{s}} U_{s 1}
$$

From (9) we can see that in same period $\Delta t$, the output torque is related to the start time, the excitation time, the excitation voltage, i.e. $U_{s 1}$, the start voltage, i.e. $U_{s 2}$ and the start angle $\theta$.All of those parameters need to be set appropriate to achieve the expected effect in start-up. The details of the underlying relationship will be discussed in next section.

The pre-excitation is implemented by a current closedloop in a DSP controller and the reference value $I_{\text {ref }}$ is calculated off-line, as in Fig.2.

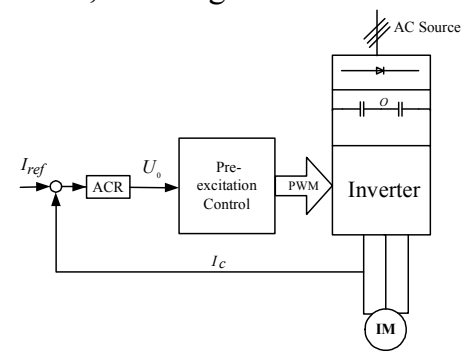

Fig.2. Block diagram of DC pre-excitation

\section{NEW ASPECTS OF DC PRE-EXCITATION AND ANALYSIS}

As mentioned in previous section, start time, excitation time, excitation voltage, start voltage and the start angle are significant parameters in a DC pre-excitation design. The appropriate setting is important for the transient process between pre-excitation and motor start moment, when the inrush current is most likely to occur.

\section{A. Start time}

The motor needs to be started immediately after the pre-excitation finished otherwise the built flux may decay. The over-excitation technique is taken for anti-decay to guarantee the flux density at the start time.

\section{B. Excitation time}

The excitation time is to ensure that the flux can be built sufficiently .Generally speaking, the excitation time should be longer than $5 \tau$.

\section{Excitation voltage}

The excitation current $I_{\text {ref }}$ is proportional to the excitation voltage $U_{s 1}$ as in (4). The density of flux is also proportional to the excitation voltage.

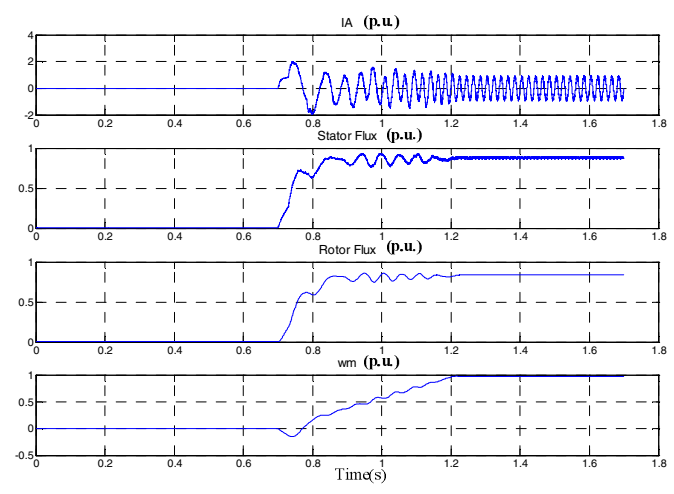

Fig.3 Simulation of motor start-up simulation with no preexcitation, $\mathrm{T}_{\text {load }}=0.5$ (p.u.)

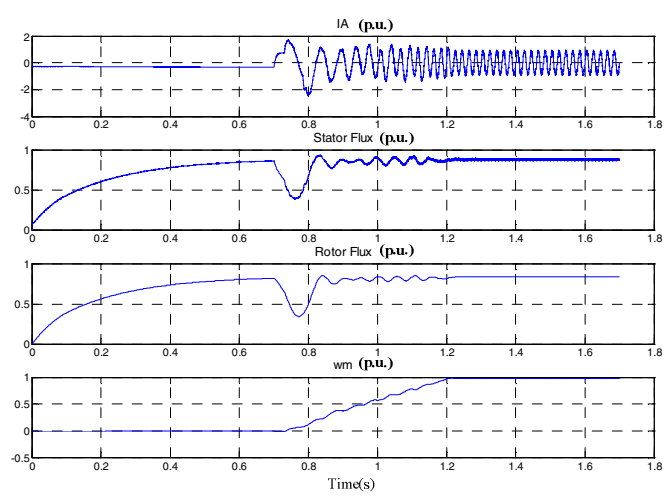

Fig.4 Simulation of motor start-up with pre-excitation $\mathrm{I}_{\text {ref }}=0.5$ (p.u.), $\mathrm{T}_{\text {load }}=0.5$ (p.u.)

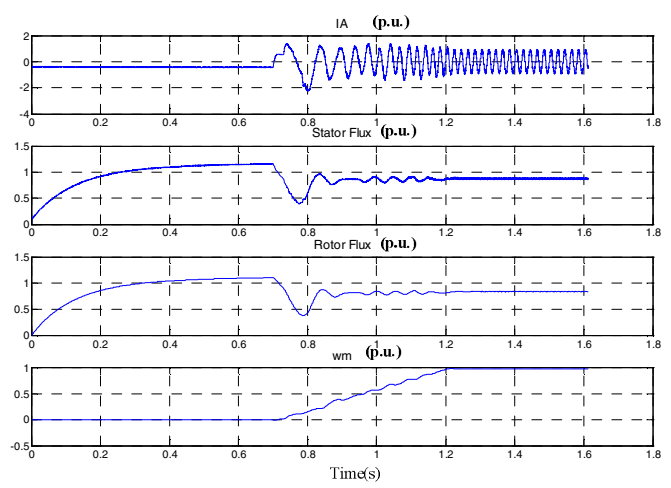

Fig.5 Simulation of motor start-up simulation with pre-excitation $\mathrm{I}_{\mathrm{ref}}=0.7$ (p.u.), $\mathrm{T}_{\text {load }}=0.5$ (p.u.) 
All of the parameter are showed in p.u. units. From Fig. 3 to Fig. 5 we can see that and the effect of preexcitation is obvious and the current is depressed with increasing of the excitation current $\mathrm{I}_{\text {ref }}$.

\section{Start voltage}

In open-loop control systems, different start curves are pre-designed to serve for different types of load. The start process following DC pre-excitation is important for inrush current depressing. It is needed to design the start curve in a suitable model for DC pre-excitation since the start curve produces the start voltage $\mathrm{U}_{\mathrm{s} 2}$ in (9).

\section{E. Start Angle}

In theory, the maximum torque can be obtained when the start angle is $90^{\circ}$. But due to the nonlinear and asymmetry characteristic of motor parameter in threephase induction motor, the optimal angle is near $90^{\circ}$ and the optimal angle can be adjusted by experiment tests for different plants.

\section{IMPROVEMENT FOR CURRENT CONTROL IN DC PRE- EXCITATION}

All of the terms above is deduced with respect to the theory of DC pre-excitation. The importance of those terms has been emphasized, but those are not all of the aspects about the implementation of DC pre-excitation, especially in the real workshop. Since the pre-excitation process is implemented based on power electronics equipment, the nonlinear characteristic of the power electronics devices and EMI in the real workshop may affect the excitation current control[7].

The restrictions in output voltage due to the non-ideal elements in switch devices are common problems in power electronics system. One of them is the minimum pulse width limitation. The existence of minimum pulse width may reduce the linear controllable area.In DC preexcitation the flux is controlled at a constant level via current closed-loop. Table I contains parameters for a motor drive system.

In the $160 \mathrm{~kW}$ motor drive system, the minimum pulse width is set at $2.4 \mu \mathrm{s}$, the minimum duty ratio in phase PWM voltage is $0.768 \%$ and the minimum controllable current is about $340 \mathrm{~A}$, which is higher than the maximum reference value: 309A. That means the current controllability gets weakened due to the existence of minimum pulse width.

TABLE. I THE PARAMETERS FOR 160KW ASD SYSTEM

The solution is to design a low-rate structure for DC pre-excitation with decreased switch frequency and calculation speed to meet the required controllability and shrink the uncontrollable current range caused by minimum pulse width limitation. When the switch frequency and calculation speed are modified to $1.6 \mathrm{k} \mathrm{Hz}$ and the minimum controllable current will decrease to $170 \mathrm{~A}$. It should to be noted that the enlarged discrete time in the low-rate structure may deteriorate the system robustness. Fig. 6 shows the abrupt current rise caused by an error output voltage, which is related to the EMI in the current sampling in $3 \mathrm{~kW}$ ASD system. Thus the low-rate structure must be equipped with anti-interference techniques to enhance the robustness.

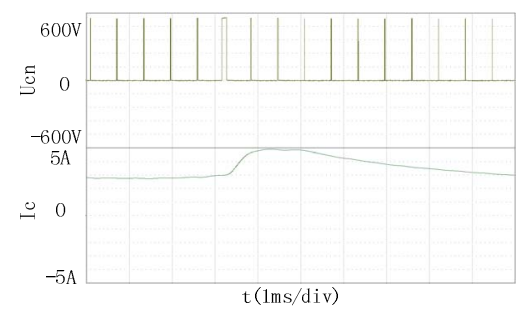

Fig.6 Current jump in DC pre-excitation in 3kW motor

As shown in Fig.6, there is a current jump in $I_{c}$. It is imperil to the switch devices when it happens in higher power system since the $\frac{d i}{d t}$ value is large and may damage the switch devices. Based on analyses of the pulse array, it can be found that the current jump is accomplished with an error pulse in $U_{C N}$ i.e. the pulse width in $U_{C N}$ jumps to the upper limit. The error pulse is caused by the error in current sampling for $I_{c}$.

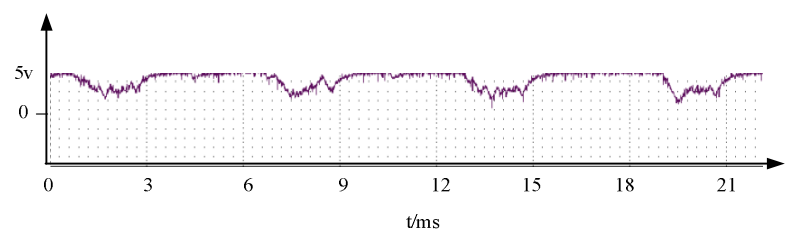

Fig.7.Errors in current sampling

A test is designed to validate the conclusion. The input analog voltage is kept at a constant DC level and the result of ADC is output through DAC. As shown in Fig.7, some sampling data by ADC jumps to an error value with large amplitude.

Different software method may be designed for shielding EMI, including: digital filtering, increasing the sampling frequency and excluding the error data by a criterion. Because the amplitude of error value is much larger than nominal value, the digital filtering works bad and by experiment test, the error sampling occurs irrespective of the sampling frequency.

A software method to shield EMI in DC pre-excitation according to the character of the error data can be applied in system.

\begin{tabular}{ccl}
\hline Rated motor voltage & Rated motor current & Stator resistance \\
\hline $380 \mathrm{~V}$ & $280 \mathrm{~A}$ & $0.007 \Omega^{1}$ \\
\hline Switch frequency & Minimum pulse width & $\begin{array}{l}\text { Maximum excitation } \\
\text { current }\end{array}$ \\
\hline $3.2 \mathrm{kHz}$ & $2.4 \mu \mathrm{s}$ & $309 \mathrm{~A}(0.9 \mathrm{pu})$ \\
\hline
\end{tabular}




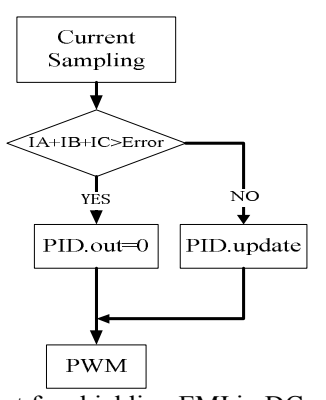

Fig.8. The flow chart for shielding EMI in DC pre-excitation

In the shielding process, the error data from current sampling is marked and filtered by a criterion and such error data would not accumulate in PID algorithm. The type of criterion needs to be designed properly in accordance with actual situation otherwise some error may not be filtered or the correct data may be confused then the normal operation may be affected.

In this case the error data occur simultaneously on three phases, and all of them jump to the value which is far away from the real value so the criterion is designed in the form of summing of current sampling values. As shown in Fig.9 the current jump is eliminated and the system reliability is improved.

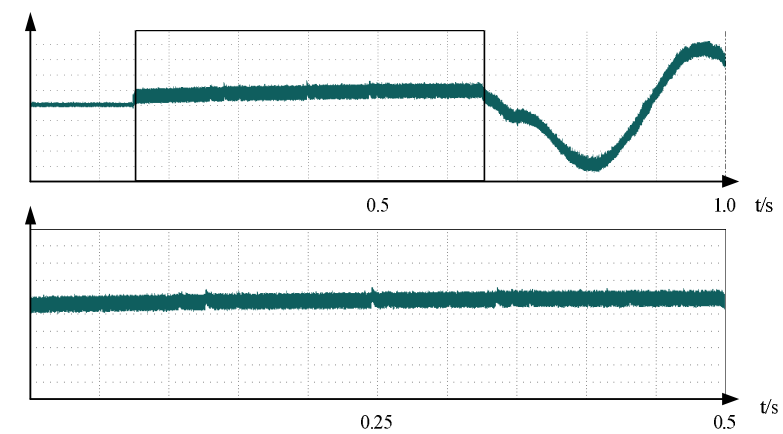

(a)

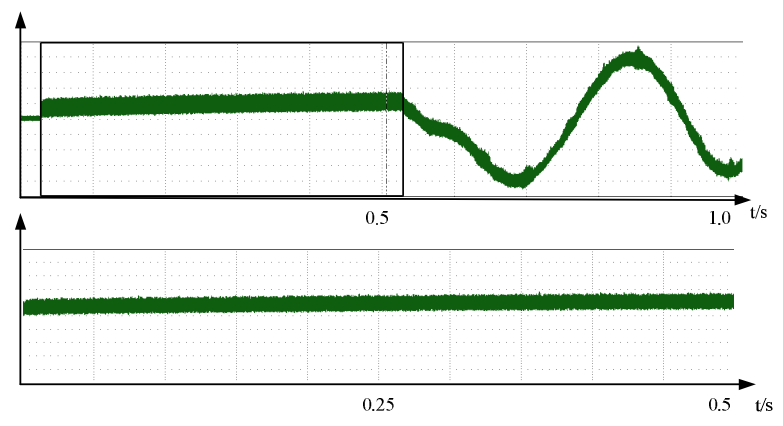

(b)

Fig.9 Effect of EMI shielding in DC pre-excitation in $3 \mathrm{~kW}$ motor (a) with EMI shielding (b) without EMI shielding

\section{EXPERIMENT RESULTS}

The experiments aim to verify the feasibility and effectiveness of the proposed approaches for the ASD system covering $55 \mathrm{~kW}-315 \mathrm{~kW}$ levels. The pre-excitation is implemented by a current closed-loop in TMS320F2812 .The experiments are conducted on 55kW and $160 \mathrm{~kW}$ ASD systems. The rated parameters of the three-phase induction motor are shown in Table II .
TABLE II

THE PARAMETER OF MOTOR

\begin{tabular}{|c|c|c|c|c|}
\hline Power & Us(rms) & Is(rms) & Poles & Torque \\
\hline $55 \mathrm{KW}$ & $380 \mathrm{~V}$ & $105 \mathrm{~A}$ & 4 & $350 \mathrm{~N} \cdot \mathrm{M}$ \\
\hline $160 \mathrm{KW}$ & $380 \mathrm{~V}$ & $280 \mathrm{~A}$ & 4 & $1018.1 \mathrm{~N} \cdot \mathrm{M}$ \\
\hline
\end{tabular}

The induction motor works in VVVF mode and is connected to a DC motor which works as a load. The DC motor is controlled by 6RA70 to produce different types of load.

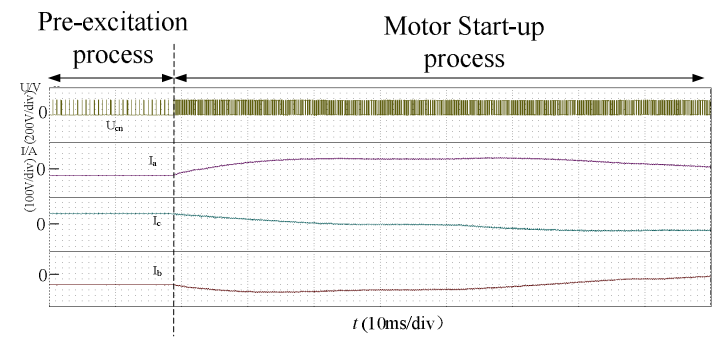

Fig.10 The voltage and current in $160 \mathrm{~kW}$ motor start-up with preexcitation

Fig.10 depicted that two switch modes exist during the process. The switch frequency in the former one is lower than in the later one. The pre-excitation current is controlled at $227 \mathrm{~A}(0.7$ p.u. $)$ stably and no current abrupt rise occurs.

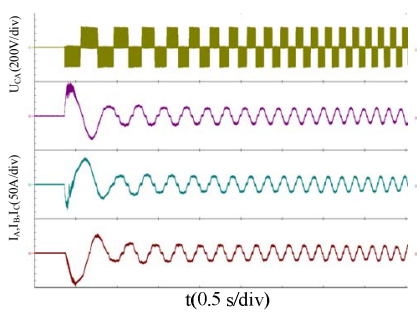

(a)

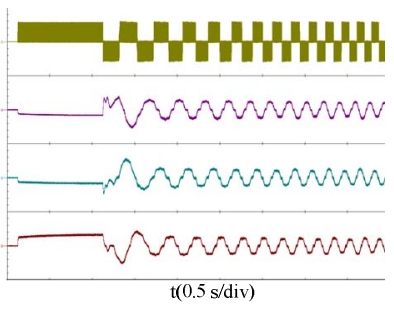

(b)
Fig.11. Comparison of 55kW motor start-up standstill with non-load (a) with DC pre-excitation (b) without DC pre-excitation.

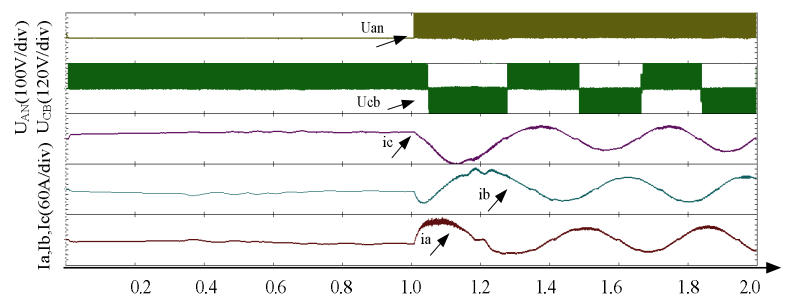

Fig.12. Starting curve of $55 \mathrm{~kW}$ motor from standstill with rated loads

As shown in Fig.11, non-load, the maximum peak during current value is $255 \mathrm{~A}$, when incorporated with DC pre-excitation the maximum peak current value is $150 \mathrm{~A}$. The inrush current is reduced by $39 \%$. As shown in Fig.12, the maximum value of $I_{e}$ in start-up with nonload is $200 \mathrm{~A}$ (peak value) and The maximum value of $I_{f}$ in start-up with full load is $300 \mathrm{~A}$ (peak value). $I_{e} I_{f}<2 * I_{s}=363 \mathrm{~A}$. While the maximum value may be larger than 4-6 times of rated current value without the proposed DC pre-excitation algorithm. Experimental results validate the effectiveness of the proposed design method.

\section{CONCLUSIONS}

DC pre-excitation is one of the effective solutions for increasing the starting torque and depressing the starting 
inrush current. However the effect of DC pre-excitation would be discounted if it could not be compatible with the induction motor drive control system. The paper analyzed the start time, excitation time, excitation voltage, start voltage and the start angle. In addition, other factors beyond the theory is also discussed and a method for EMI shielding in DC pre-excitation to improve the current control reliability is proposed, which is not presented in previous literatures. Experimental results in a $55 \mathrm{~kW}$ and $160 \mathrm{~kW}$ ASD system validate the effectiveness of the proposed design method.

\section{REFERENCES}

[1] Li, Jingchuan,Li Qingfu,Zhao Guang"Simulation of inverter induction motor drive systems using tensor method," Proceedings of the Chinese Society of Electrical Engineering,vol.19,1999

[2] GE Baojun; LI Yuling; "Study of process of starting pumped storage machine by static frequency converter with field current controlled", Electric Machines and Control,vol.7,pp.187-190,2003.

[3] Guan Hui, Zhao Zhengming, Mengshuo "Optimal design for inverter-driven induction motors," Proceedings of the CSEE,vol.24,pp.194-199,2004

[4] Juhasz G,Halasz S,Veszpremi K.New aspects of a direct torque controlled induction motor drive[C].Proceedings of IEEE International Conference onIndustrial Technology ,Goa,India, 2000.

[5] Bai Hua, Zhao Zhengming, Hu Xian, Chen, Xin. "Experimental analysis of DC pre-excitation for 3-level inverter-motor system,"Proceedings of the CSEE,vol.26,pp.159-163,2006.

[6] Shi Tingna; Wu Shuguang; Fang Youtong; Chen Wei; Xia Changliang. "Reasearch on sensorless PM brushless DC motor starting control," Proceedings of the Chinese Society of Electrical Engineering, vol.29,p111-116,2009

[7] Sideng $\mathrm{Hu}$;Zhengming Zhao; "A novel modbus RTUbased communication system for adjustable speed drives," IEEE Vehicle Power and Propulsion Conference, VPPC, 2008 